\title{
TRACKING AND MONITORING OF TAGGED OBJECTS EMPLOYING PARTICLE SWARM OPTIMIZATION ALGORITHM IN A DEPARTMENTAL STORE
}

\author{
INDRAJIT BHATTACHARYA ${ }^{1}$, KAUSIK DAS SHARMA ${ }^{1}$ AND UTTAM KUMAR ROY ${ }^{2}$ \\ ${ }^{1}$ Kalyani Government Engineering College, Kalyani, Nadia, INDIA \\ ${ }^{2}$ Department of Information Technology, Jadavpur University, INDIA
}

E-mails:indra51276@gmail.com,kaushikdassharma@yahoo.com,u_roy@it.jusl.ac.in

\begin{abstract}
The present paper proposes a departmental store automation system based on Radio Frequency Identification (RFID) technology and Particle Swarm Optimization (PSO) algorithm. The items in the departmental store spanned over different sections and in multiple floors, are tagged with passive RFID tags. The floor is divided into number of zones depending on different types of items that are placed in their respective racks. Each of the zones is placed with one RFID reader, which constantly monitors the items in their zone and periodically sends that information to the application. The problem of systematic periodic monitoring of the store is addressed in this application so that the locations, distributions and demands of every item in the store can be invigilated with intelligence. The proposed application is successfully demonstrated on a simulated case study.
\end{abstract}

KEY WORDS: Radio Frequency Identification (RFID), Particle Swarm Optimization (PSO), Departmental Store Automation System.

\section{INTRODUCTION}

A departmental store has different types of items enter onto the store and placed in its proper rack. The items are tagged using passive RFID tags and each of them receives a unique identity number. The readers are placed in the strategic positions in the store to automate the identification process and send the information of the items in its communication range. Here we have used the passive tags for minimizing the cost of the system. The radio range of the reader is 50 meters. Hence a number of readers are required to monitor the items spanned over entire departmental store. The readers gather information of the items in their zones and send that information periodically to the application for taking appropriate decisions.

This paper presents the design, development and implementation of a RFID based departmental store to periodically monitor the items and to identify the most and least demanded item list using PSO algorithm that in turn would be useful for theft detection and stock updates for that store. 
The RFID technology provides accurate information on the number of items, their locations in the store and their distributions, while the proposed algorithm manages the information, better services and information related to demand of items in the store.

Swarm Intelligence (SI) is an Artificial Intelligence (AI) technique involving the study of cooperative behavior in decentralized systems. Such systems are made up by a population of individuals interacting locally with one another and with their environment. There is no centralized control dictating the behavior of the individuals, local interactions among the individuals often cause a global pattern to emerge. SI refers to the problem solving behavior that emerges from the interactions between the individuals and the computational swarm intelligence refers to algorithmic models of such behaviors. These algorithmic models have shown to be able to adjust well in changing environments and are greatly flexible and robust. The last decade has shown a rapid growing research interests in SI, particularly on the most popular SI paradigm namely Particle Swarm Intelligence (PSO).

PSO is a stochastic, population-based optimization algorithm introduced in 1995 by James Kennedy and Russell C. Eberhart [1,2]. Many variants of PSO have been developed since, including constrained, multi objective, and discrete or combinatorial versions and applications have been developed using PSO in many fields.

\section{RELATED WORK}

The first practical application of PSO was in the field of neural network training and was reported together with the algorithm itself $[3,4,5,6]$. Many more areas of application have been explored ever since, including telecommunications, control, data mining, design, combinatorial optimization, power systems, signal processing, and many others. To date, there are hundreds of publications reporting applications of particle swarm optimization algorithms [4]. In [7] the PSO algorithm was applied effectively for locating an intruder in a wireless ZigBee based network. Although PSO has been used mainly to solve unconstrained, single-objective optimization problems, PSO algorithms have been developed to solve constrained problems, multi-objective optimization problems, problems with dynamically changing landscapes, and to find multiple solutions $[8,9,10]$. In [11] the PSO algorithm was effectively implemented to design a fuzzy controller to control the robot movement. In [12] the PSO algorithm was successfully implemented to optimally place RFID readers in a RFID network.

\section{SYSTEM DESCRIPTION}

The whole premise of the departmental store is divided into four zones (Fig.1), where each zone is equipped with a passive RFID reader with a built-in ZigBee transceiver. In that premise there is an entry point and an exit point equipped with RFID Reader.

In each zone there are several racks, which are used to store the items of the same types. 


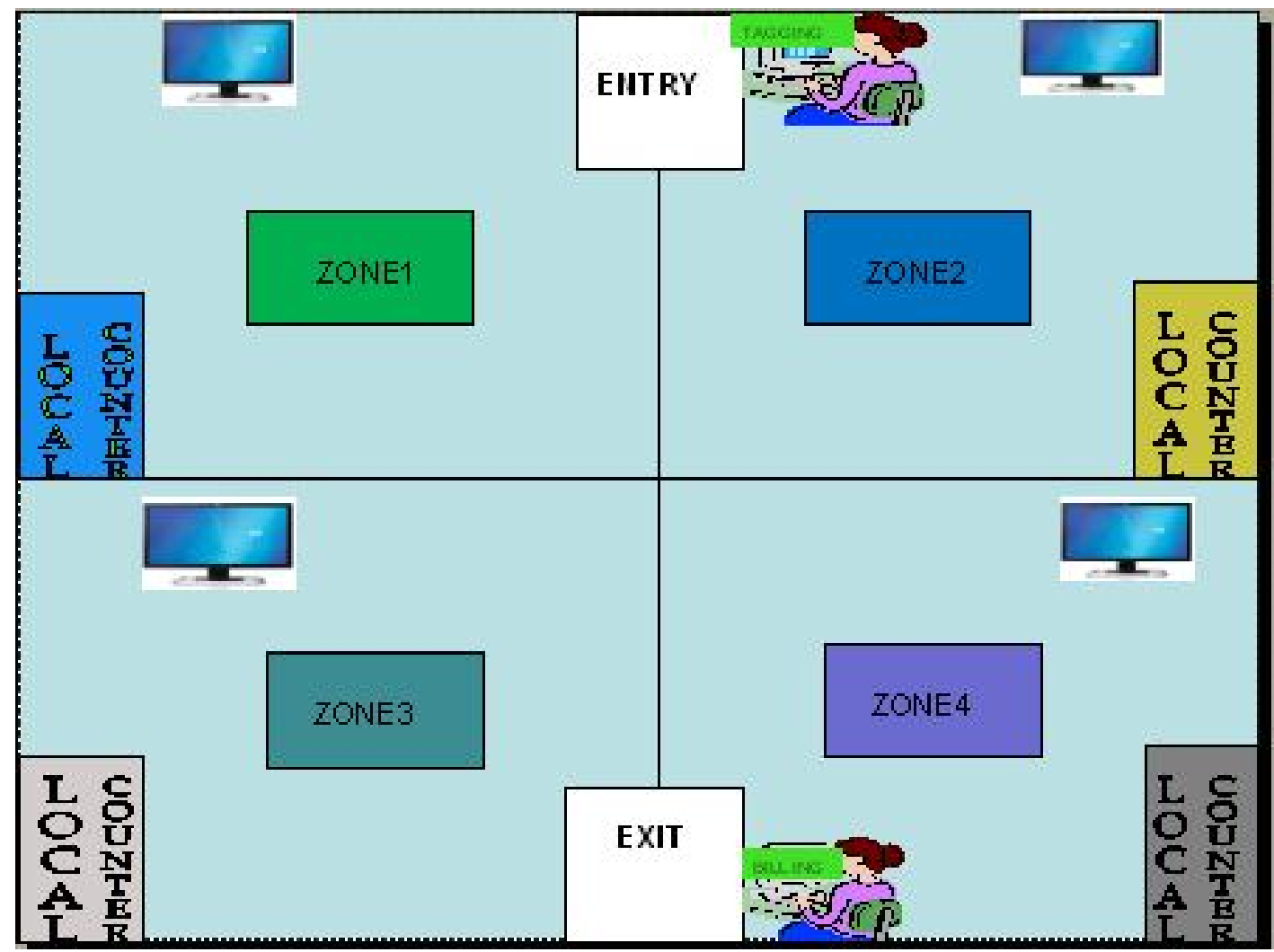

Fig. 1: Layout of the system.

While entering to the store each item will be attached with a Passive RFID tag and each tag will contain information regarding their identity, which can be described in terms of a record of 5-tuples (Type of the Product, Sub typeOf the Product, Product-id, Position, Date).

The tagging has been done in the following way:

$$
\text { TAG }=[\text { TypeOfProduct } \mid \text { Subtype } \mid \text { Product-ID } \mid \text { Position } \mid \text { Date }]
$$

Each tagged item should have five fields. They are:

a) Type of Products: Such as Garment, Utensils, Food, Electronics goods, etc. Here domain of this field is Alphabets of English language. For example, All Garments products should be tagged by character ' $G$ ' and so on.

b) Subtype of products: For each product type there may be sub product types such as electronics good may be of TV, VCD, etc. The domain of this field is Alphabets of English language.

c) Product-ID: This field denotes a unique number, which is tagged to each product used for identity of the product. The domain of this field is string of 0's and 1's of 16bits long. 
d) Position: The domain of Position field is simply positive integer. Position field once again sub-categorized into two fields, which are xcoordinate and y-coordinate. According to the Type and Subtype of the item, all items will be stored to their corresponding position (Basically in rack kept in each zone).

e) Date: This field is of the pattern dd-mm-yyyy. Whenever an item enters to the store, it is tagged with current date.

After the items are tagged, they are placed to their corresponding position in the rack sequentially according as their Type and Subtype.

Reader of each zone is capable of monitoring their respective items. So the Reader is capable of detecting the presence of all items within its vicinity at that particular zone. The presence or disappearance of a tagged item from a particular zone is automatically reported to a server located in the command room. This server is where the database is kept and it is also where the application software is run. The total database of the items with others is stored in the server. Communication between the RFID readers and the server is through the built-in ZigBee transceiver and the self-configuring network topology.

There are four counters one for each zone. The customers first take their selected items to be purchased to the respective counters. That is, they should carry the Garments items to the Garment-counter and Electronic Goods to the Electronic Goods-counter and so on.

From each counter the selected items are sent to the exit point where the BILLING is done. It should be taken care of the fact that the interference among the items in different zones should be minimized.

From the automation application point of view, we add another attribute which is called VALID_TIME of products or items. VALID_TIME is actually Threshold time for each product. Which signifies that products can be kept in the store for a predefined period of time and after that product should be replaced or renewed. The system is capable of tracking the records of each product and will give a signal if the VALID_TIME of any product is expired. This VALID_TIME can be set by the shop-owner.

\section{AUTOMATION SYSTEM GOALS}

The system can help a departmental store-owner to meet his various needs. For instance, the system will provide the information to the owner after fixed time of interval which products were sold most and which is not. The owner need not take this review manually from the store.

1) The owner may be interested about which products are of high demands to the customers. For this query, owner needs not to approach to the customers because the system can generate this information whenever it is requested.

2) Since the automation system is tracking all the objects every time so theft can be prevented using this system. 
The system can also be used to manage stock in an efficient way by virtue of knowing the demands of the items in the store periodically.

\section{PARTICLE SWARM OPTIMIZATION - A BRIEF DESCRIPTION}

In 1995 Particle Swarm Optimization (PSO), a population based swarm algorithm was developed by Kennedy and Eberhart [1][2]. In the PSO computational algorithm, population dynamics simulates bio-inspired behavior i.e. a "bird flock's" or "school of fish" behavior which involves sharing of information and allows particles to take profit from the discoveries and previous experience of all other particles during the search of food. Each particle in PSO has a randomized velocity vector $(\underline{v})$ associated to it, which moves through the search space. Each particle in PSO keeps track of its coordinates in the solution space, which are associated with the best solution (fitness) it has achieved so far.

This value is called $\underline{p}$ (personal best position vector). Another best value that is tracked by the global version of the particle swarm optimizer is the overall best value (fitness). Its location, called $\underline{g}$ (global best position vector), is obtained among all the particles in the population. The past best position of the particle itself and the best overall position in the entire swarm are employed to obtain new position vector $(\underline{s})$ for the particle in quest to minimize (or maximize) the fitness.

At each time step t the velocity of the particle is updated and the particle is moved to a new position. This new position is calculated as the sum of the previous position and the new velocity:

$$
\underline{S}_{t+1}=\underline{s}_{t}+\underline{v}_{t+1}
$$

The update of the velocity from the previous velocity to the new velocity is determined as:

$$
\underline{v}_{t+1}=\omega \cdot \underline{v}_{t}+r_{1} \cdot c_{1}\left(\underline{p}_{t}-\underline{s}_{t}\right)+r_{2} \cdot c_{2}\left(\underline{g}_{t}-\underline{s}_{t}\right)
$$

The PSO concept consists, in each time step, of changing the velocity of each particle flying towards its personal best and global best location with an inertia weight $\omega$, which controls the magnitude of the old velocity in the calculation of new velocity as:

$$
w_{t}=\frac{\left(\text { iter }_{\max }-t\right) *\left(w_{\text {start }}-w_{\text {end }}\right)}{\text { iter } r_{\max }}+w_{\text {end }}
$$

where itermax is the maximum number of iterations of PSO. Normally wt is reduced linearly from wstart to wend throughout the PSO generation. A typical combination is $w_{\text {start }}=0.9$ and $w_{\text {end }}=0.4$.

The velocity is weighted by random terms, with separate random numbers ( $r l$ and $r 2$ ) being generated for velocities towards personal best and global best locations respectively. 
$c 1$ and $c 2$ are the social parameters used for the learning purpose in the optimization technique.

Some research approaches investigated the application of constriction coefficients and inertia weights. There are numerous techniques for preventing premature convergence. Many variations on the social network topology, parameter-free, fully adaptive swarms, and some highly simplified models have been created. The algorithm has been analyzed as a dynamical system, and has been used in hundreds of engineering applications; it is used to compose music, to model markets and organizations, and in art installations.

\section{IMPLEMENTATION OF PSO BASED RFID TRACKING SYSTEM}

The main focus of our work is to implement an automatic object monitoring system using PSO algorithm and RFID technology [13, 7]. Here RFID system is being used for tagging and automatic tracking the items of a departmental store and PSO is used to modify the movements of items with in the whole premise of departmental store. The main objectives of our system are:

1) Develop an automated object monitoring system.

2) Provide security of objects belonging to the system.

3) To minimize the zonal interference among the objects moving in the system.

4) To sort objects according to their movements. More precisely, to find which objects are much demanded and which are less demanded.

5) To provide a quick review of current stock.

6) Make the authority alert if there is any mismatch of distribution of objects (misplacing of items with respect to their arrangement).

7) Identifying those objects which are achieved goal mostly make the authority aware about the demands of customers.

The functional description of the system is depicted in the following flowchart: 


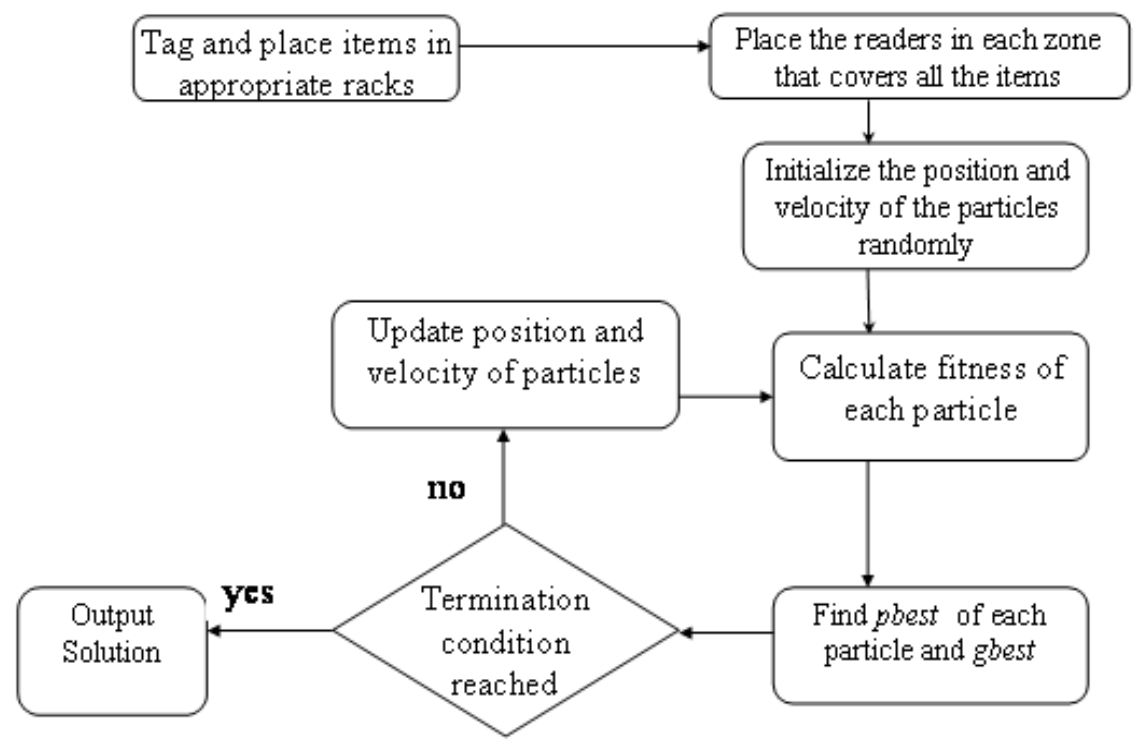

Fig. 2: Flowchart showing the functionality of the system.

With analogy of PSO the exit point is where the food is located. All members of the group will converge to these points after a period of time. Therefore the goal our algorithm is to identify the less demanded and most demanded items from a group of items are based on PSO. At the entry point we group the items depending on the product type and subtype and are monitored periodically with in the store. The readers will report the positions of the items periodically. Based on the location information the system can work out and record their movements as well as their stay in the zones of the store.

These data are used for comparison with each member; those members with the highest discrepancy are identified using an appropriate fitness function from those data can be classified as less-demanded items. Those items of the store are either need to be replaced or renewed.

In the PSO implementation $[14,15,16,17]$ we have considered a problem space, which is divided into four zones. There is one entry point and one exit point. The particles are entered to the store through the particular entry point and then the actual tagging is done. In the tagging procedure we have considered the five attributes for each item. They are the product type, subtype, product-id, (initial x-coordinate, initial y-coordinate), and the date of entry. The unique attribute of an item is the product-id. The items are mapped into a 2D space and will obtain its position (x,y) depending upon it's type and subtype and that is going to considered as the initial position of the particles in PSO. In each zone there is a local counter. The particle after being sold is first moved to the local counter and then from the local counter those are moved towards the exit section where actually the billing is done. For the moving of the particle from the local counter to the exit point, we have considered that the zonal interference should be minimized. In each zone there is a reader, which is monitoring the location and movement of the particle of the specified zone. The reader's have the monitoring power for a specified radius. There are also two RFID 
readers who are placed in the entry point and the exit point. So the system can get the locations of the items periodically from the readers. Each particle has the particular lifetime. The user can easily change the lifetime limit for the particle. The particles are considered to be volume less.

The function location is used to search and place the new item in the proper position in the rack of the store. The function also fixes the position of the item in a 2D space depending on the product type and subtype and then they are treated as particles. In this way all the items are get placed in the proper rack of the store in the specified zones.

A 2D array is used to check the availability of the position in the rack. Initially all positions are initialized with the value zero. When a particle is placed in the shelf its corresponding location is updated with the value one. The function location first checks the array whether the corresponding location is one or zero. If it is zero then it places the particle on the location, otherwise it checks for the availability at the next position.

As it is known in case of PSO algorithm, the particle best position is determined by the fitness function in each iteration. The fitness function has been calculated by function W. In our work the fitness function is used to keep track of those particles, which are less demanded and does not converge to the solution after a predetermined number of iterations. The user has to fix one specific lifetime for each product. When the particle's date difference (i.e. difference of entry date from the current date) exceeds the specified threshold value then it is identified as less demanded particle.

In our implementation the inertia weight was varied from 0.9 to 0.4 and $c 1, c 2$ are the two positive constants set to 0.1. Applying PSO algorithm the position and velocity of the particles are updated for a specified number of iterations and the particles move towards the destination.

\section{SIMULATION RESULTS}

In PSO implementation we have considered a problem space, which is the area covered by the departmental store. We have carried out our simulation in a 500x300 pixel area. The whole area is divided into four equal sized zones. Each zone is placed with artificial racks. We have assumed that items can be placed at pixels within the racks only. The transmission range of the readers is set to 20 meters and readers are placed in the appropriate position of each zone to periodic monitor each of the items in that zone. We have carried out experiments over five items and performed number of iterations on PSO algorithm varying the inertia weights of the particles from 0.9 to 0.4 in each iteration and applied fitness function at each step. The result in figure 3 shows that two particles (denoted as item1 and item5) has been reached to the destination within 100 iterations, and rest of three does not converge to the solution after 200 iterations. This signifies that the items 2,3 and 4 (denoted by the particles in PSO implementation) are less demanded items and can be added to the list of less demanded item list which need to be replaced in the store. Similarly the item 1 and item 5 can be identified as two most demanded items in the shop. 


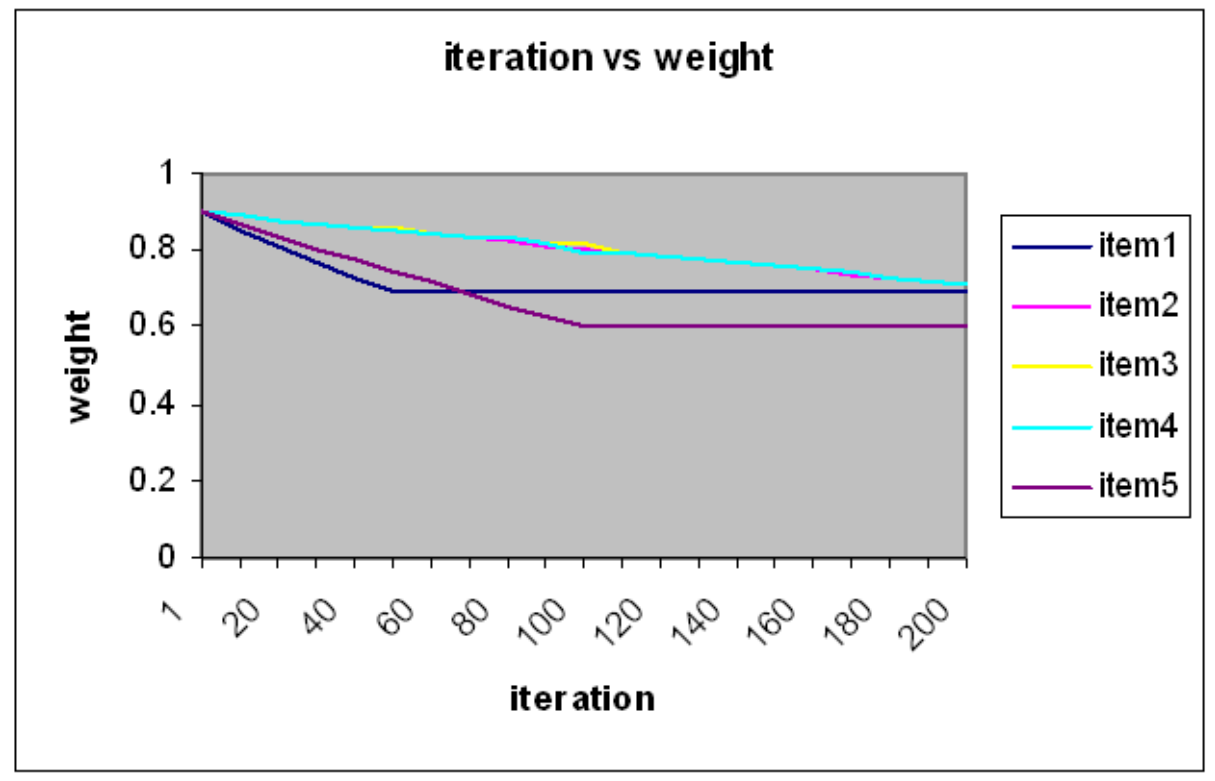

Fig. 3: Iterations and inertia weight(w) assigned.

From Fig. 4 we can determine the demands of the items in the departmental store. Here we have considered product type as Electronics(E), Garments $(\mathrm{G})$, Foods $(\mathrm{F})$ and Others $(\mathrm{O})$. This information can be used by the shop owner for order placing and stock updates.

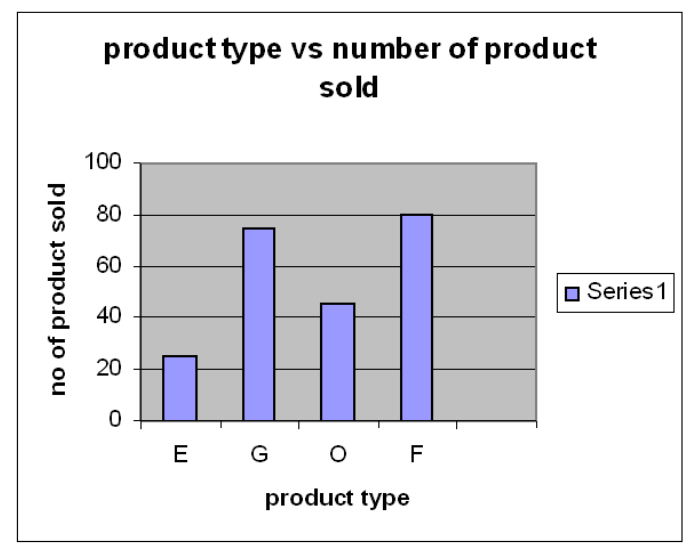

Fig. 4: Chart showing demands of items according as their types.

From Fig. 5 it can be concluded that before the iteration number 193, no particles are converged but after that with the increase of iterations particles started to converge and as number of iteration increases the convergence rate also increases. It is obvious that convergence rate will never be $100 \%$ from our experimental graph as not all the particles would be eventually converge. Our aim here is to identify those particles and hence the items (that information can be found from the database) which do not reach to the 
destination can be considered as less demanded items. Here convergence rate has been calculated using following formula:

Convergence rate $=($ no. of particles reaches the destination / total no. of particles $) * 100$

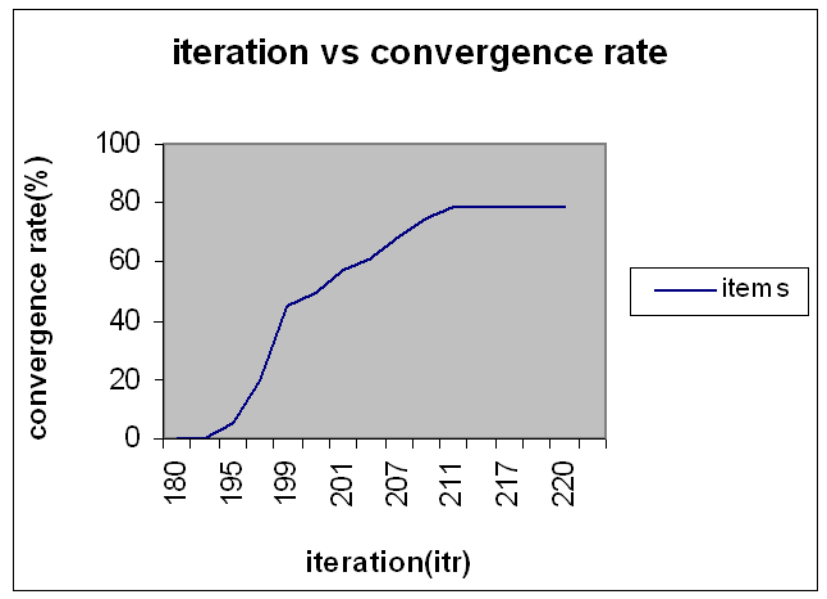

Fig. 5: Iterations and convergence rate.

\section{CONCLUSIONS}

In this paper the problem of systematic periodic monitoring of the store is addressed so that the locations, distributions and demands of every item in the store can be invigilated with intelligence. Applying PSO algorithm in the application it has been possible to identify the less demanded items in the store which need to be replaced. The backbone RFID network monitors the store periodically and sends the item information to the application on which the PSO algorithm works. The demand analysis of the items of the store can be done by the system in an efficient manner which may be useful for stock management of the store.

\section{REFERENCES}

[1] R. C. Eberhart and J. Kennedy, "A new optimizer using particle swarm theory," Proc. 6th Int. Symp. Micromachine Human Sci., Nagoya, Japan, pp 39-43, 1995.

[2] J. Kennedy and R. C. Eberhart, "Particle swarm optimization," Proc. IEEE Int. Conf. Neural Networks, vol. 4, pp. 1942-1948, 1995.

[3] J.Kennedy "The Particle swarm: social adaptation of knowledge" Proc. IEEE Int. Conf. on Evolutionary Computation (Indianapolis, Indiana), IEEE Service Center, Piscataway, NJ, pp. 303-308, 1997.

[4] R.C. Eberhart, Y.H. Shi "Comparison between genetic algorithms and particle swarm optimization" Annual Conf. on Evolutionary Programming, San Diego, 1998.

[5] Qiang Guan, Yu Liu, Yiping Yang, Wesshueg Yu "Genetic Approach for Network Planning in RFID Systems" Proceedings of the sixth Int. Conf. on Intelligent Systems -ISDA, 2006. 
[6] R. Poli "Analysis of the publications on the applications of particle swarm optimization" Journal of Artificial Evolution and Applications, Article ID 685175, 10 pages, 2008.

[7] M.S. Rodrigo, Carlos L.S.S. Sobrinho, S.Araujo, Farias Rubem "Particle Swarm Optimization Applied for Locating an Intruder by an Ultra-Wideband Radar Network" Federal University of Para, Brazil.

[8] Y.H.Shi, R.C. Eberhart "A modified Particle swarm optimizer. IEEE Int. Conf. on Evolutionary Computation” Anchorage, Alaska, May 4-9, 1998.

[9] P. J. Angeline "Evolutionary Optimization versus particle swarm optimization: philosophy and performance difference" Annual Conf. on Evolutionary Programming, San Diego, 1998.

[10] A.P.Engelbrecht "Fundamentals of Computational Swarm Intelligence" Willey, 2005.

[11] K. Das Sharma, A. Chatterjee and A. Rakshit "A Hybrid Approach for Design of Stable Adaptive Fuzzy Controllers Employing Lyapunov Theory and Particle Swarm Optimization" IEEE Transactions on Fuzzy Systems, vol-17, no.-2, pp-329-342, April, 2009.

[12] I. Bhattacharya, U.K.Roy "Optimal Placement of Readers in an RFID Network Using Particle Swarm Algorithm", IJCNC, vol-2, no-6, pp-225-234, November 2010.

[13] Y.M. Siu, W.S.Chan, K.K. Soo, C.W.Leung, S.M. Law "Exhibition Security and Safety System Using RFID Technology and a modified Particle Swarm optimization Algorithm” Asia-Pacific Microwave Conf. APMC, 2008.

[14] P.J.Angeline "Using selection to improve particle swarm optimization" IEEE Int. Conf. on Evolutionary Computation, Anchorage, Alaska, May 4-9, 1998.

[15] Y.H.Shi, R.C. Eberhart "Parameter selection in particle swarm optimization" Annual Conf. on Evolutionary Programming, San Diego, March 1998.

[16] Wail Gueaieb and Md. Suruz Miah "Mobile Robot Navigation using PSO and Noisy RFID communication" IEEE Int. Conf. on Computational Intelligence for Measurement Systems and Applications, 14-16 July, 2008.

[17] Hui Zhu, Syahrulanuar Ngah, Ying Xu, Yuji Tanabe, Takaaki Baba "A Random Time-Varying PSO for local positioning systems”. IJCSNS, Vol 8. No. 6, June, 2008. 
